\title{
Epidermis-restricted expression of zebrafish cytokeratin II is controlled by a $-141 /+85$ minimal promoter, and cassette $-141 /-111$ is essential for driving the tissue specificity
}

Received November 20, 2005; accepted in revised form January 17, 2006

\begin{abstract}
We isolated a $2.3 \mathrm{~kb}$ DNA segment from the upstream region of the zebrafish cytokeratin II (zfCKII) gene. Transgenic embryos, produced by using a series of $5^{\prime}$ deletions linked to the red fluorescent protein (RFP) reporter, showed that the $-141 /+85$ segment of zfCKII directed RFP expression in epidermal cells, whereas the $-111 /+85$ segment did not. When $-141 /-111$ was deleted from $-355 /+85$ and microinjected into onecelled embryos, no fluorescence was observed at later stages, indicating that the $-141 /-111$ segment is required for green fluorescent protein expression in epidermal cells. Furthermore, when a putative KLF-binding site at $-119 /-117$ was mutated, RFP expression rates and intensities were reduced dramatically, although still observed, suggesting that $-119 /-117$ within -141 / -111 is a key cis-element for controlling epidermis-specific expression of the zfCKII gene. Finally, we generated a zebrafish transgenic line, $\operatorname{Tg}(\mathrm{zfCKII}(2.3)$ :RFP), which carries an upstream $2.3 \mathrm{~kb}$ regulatory region of the zfCKII gene fused with RFP. The expression pattern in the epidermal cells of $\operatorname{Tg}(\mathrm{zfCKII}(2.3)$ :RFP) fish recapit-
\end{abstract}

\footnotetext{
Yun-Hsin Wang* · Jun-Hung Lu · Yu-Ju Lin

Huai-Jen Tsai $(\bowtie)$

Institute of Molecular and Cellular Biology

National Taiwan University

No. 1, Sec. 4, Roosevelt Road

Taipei 106, Taiwan

Tel: 886-2-3366-2487

Fax: 886-2-2363-8483

E-mail: hjtsai@ntu.edu.tw
}

Yau-Hung Chen* . Min-Yen Chang

Graduate Institute of Life Sciences

Tamkang University,

Tamsui, Taiwan

*Equal contribution. ulated that of the endogenous gene. F2 embryos derived from $\operatorname{Tg}(\mathrm{zfCKII}(2.3)$ :RFP) males crossed with wild-type females revealed that the earliest onset of RFP expression was at the sphere stage, indicating that this transgenic approach can be used for studying zygotic expression of maternally inherited genes.

Key words cytokeratin - epidermal cells $\cdot$ germline transmission - red fluorescent protein - transgenic . zebrafish

\section{Introduction}

There are many families of cytoplasmic filament proteins. Keratin is one such family, having intermediate size $(10 \mathrm{~nm})$ and expression specifically in epithelial cells as cytoskeletal proteins. At least 30 related members in the cytokeratin family are known and are encoded by complex multiple genes. Type I keratins (K9-K20) are more acidic $(p \mathrm{I}=4-6)$, whereas type II keratins (K1-K8) are neutral or basic $(p \mathrm{I}=6-8)$. Unlike other intermediate filament (IF) proteins, cytokeratins form obligatory heteropolymers, consisting of equal numbers of type I and type II keratins (Fuchs and Weber, 1994; Klymkowsky, 1995; Rogers et al., 2004) as specific pairs, and form filaments by the coiled-coil interaction (Hatzfeld et al., 1987).

There are 19 human keratin isoforms (K1-K19), and specific pairs of keratin isoforms determine tissue identity and differentiation state. For example, human keratins K5 (type II) and K14 (type I) together form the extensive IF network of mitotically active basal cells in all stratified epithelia (Byrne et al., 1994). Expression of human keratin is restricted: E keratins are found in stratified epithelium (including epidermis), and $\mathrm{S}$ kera- 
tins are found in simple epithelium. However, in rainbow trout, both $\mathrm{E}$ and $\mathrm{S}$ keratins are expressed in intestinal simple epithelium (Markl and Franke, 1988). In zebrafish, S keratins are expressed both in stratified epithelium (including epidermis) and simple epithelium (Imboden et al., 1997). In addition, fish keratin can be found in mesenchyme-derived cells and certain nerve cells (Giordano et al., 1989, 1990), but mesenchymal tissue usually expresses vimentin in humans. This evidence indicates that fish epidermal keratin diversified independently from that of mammalian epidermal keratin. Thus, the mechanism of transcriptional regulation of the keratin in fishes should be studied.

In mammals, the regulation of keratin genes by a variety of cis-acting elements has been studied. For example, a keratinocyte-specific enhancer (the $10 \mathrm{bp} \mathrm{5'}$ ACCTGCAGGC-3') has been identified in the K14 gene (Vassar et al., 1989; Leask et al., 1990). Nuclear proteins, such as GKLF/KLF4 and Sp1, which interact with the upstream region of the human K19 keratin gene, have also been identified (Brembeck and Rustgi, 2000). Rhodes and Oshima (1998) reported that the expression of human and mouse K18 is activated by the transcription factors $c$ jun and $c$-fos, respectively, by means of an outer AP1 site in the first intron. Additionally, Popa et al. (2004) reported that the AP-2 transcription factor family is presumed to play an important role in the regulation of the keratinocyte squamous differentiation program. There is extensive knowledge about the transcriptional regulation of keratin genes in mammals; however, the mechanism of transcriptional regulation of keratin in fish has not been reported to date, although fish cytokeratin has been characterized from rainbow trout (Markl and Franke, 1988; Markl et al., 1989; Schaffeld et al., 2002a, 2002b), goldfish (Druger et al., 1992), catfish (Ainis et al., 1995), lamprey (Zaccone et al., 1995), mosquito fish (Arenas et al., 1995), common carp (Groff et al., 1997), zebrafish (Conrad et al., 1998; Chua and Lim, 2000; Schaffeld et al., 2003; Wang et al., 2006b), and shark (Schaffeld et al., 1998).

Previously, a zebrafish type II cytokeratin (zfCKII) cDNA was isolated and characterized (Chua and Lim, 2000). zfCKII was expressed uniformly in the skin, fins, scale epidermis, retina, cornea, neurons, and glial cells of the brain and spinal cord as well as in chondrocytes of the skull of the adult fish. The tissue-specific expression of zfCKII is of particular interest because of the broad diversity of zfCKII-positive epithelial cells. In the present study, a $2.3 \mathrm{~kb}$ zfCKII promoter was isolated and its function was assayed in both transient and stable transgenic lines. In stable transgenic lines, red fluorescent protein (RFP) expression faithfully reflected the expression of the endogenous zfCKII gene. Microinjections of serial deletions of the upstream segment of zfCKII fused with RFP were performed. We found that a cis-element of the DNA segment in the zfCKII gene is required for epidermal cell-specific expression of zfCKII and that a minimal region upstream of the transcription start site $(141 \mathrm{bp})$ is required for this expression restricted to epidermal cells.

\section{Materials and methods}

Experimental fish

Zebrafish AB strains (wild-type, wt) were kept under a $14 \mathrm{hr}$ light and $10 \mathrm{hr}$ dark photoperiod at approximately $28.5^{\circ} \mathrm{C}$. After fertilization, the eggs were collected and cultured in an aquarium. Embryonic cleavage and somite formation were observed with a light microscope to determine the developmental stages (Kimmel et al., 1995).

Isolation of the zfCKII upstream regulatory region

The $5^{\prime}$-flanking region of the zfCKII gene was isolated according to the instructions for the Universal GenomeWalker Kit (BD Biosciences Palo Alto, CA). Briefly, DNA was extracted from zebrafish embryos (Westerfield, 1995), digested with restriction enzymes, and ligated to a GenomeWalker adaptor to produce GenomeWalker libraries. DNA fragments containing the putative upstream region were isolated after two successive polymerase chain reaction (PCR)based DNA walking in GenomeWalker libraries. The primary PCR and the outer adapter primer (AP-1) products were obtained using the GeneAmp PCR System with five cycles of $94^{\circ} \mathrm{C}$ for $5 \mathrm{sec}$ and $72^{\circ} \mathrm{C}$ for $3 \mathrm{~min}$, followed by 30 cycles of $94^{\circ} \mathrm{C}$ for $5 \mathrm{sec}$ and $68^{\circ} \mathrm{C}$ for $3 \mathrm{~min}$, and a final extension at $68^{\circ} \mathrm{C}$ for $7 \mathrm{~min}$. For each round of genomic walking, the diluted primary PCR products served as templates for the secondary nested PCR with a nested gene-specific primer (CKIIR) and the nested adaptor primer (AP-2). The secondary PCR products were analyzed and purified from agarose gels and cloned into pGEMT easy Vector (Promega, Madison, WI) to produce pT7CKII. The primers used in the genomic walking and subsequent cloning are listed in Table 1.

\section{$5^{\prime}$-Rapid amplification of cDNA end (5'-RACE)}

The protocols of 5'-RACE are followed by the manufacturer's instructions (FirstChoice ${ }^{\mathrm{TM}}$ RLM-RACE; Ambion, Austin, TX) with minor revision. Briefly, first-strand cDNA used for 5'-RACE was performed as described before (Chen et al., 2001), and then using 5 '-RACE outer primer and CKII-271R for the first PCR, and primers CKII-117R and 5'-RACE inner primer for the nesting. Amplified DNA fragments were sub-cloned and sequenced as described above.

\section{Construction of chimeric reporter gene fusions}

The upstream segments of zfCKII and of the serial deletion derivatives were fused with the RFP reporter gene by cloning the various $5^{\prime}$ deletion fragments into the polylinker region of the vector pDsRed2.1 (Clontech, Palo Alto, CA) upstream of a promoterless RFP gene. To generate pCKII2.3K-RFP, pT7CKII was digested by $E c o$ RI and a $2.3 \mathrm{~kb}$ EcoRI-digested fragment was purified and ligated to EcoRI-digested pDsRed2.1 (Clontech). The deletion fragments of $1.7 \mathrm{~kb}, 1.2 \mathrm{~kb}, 526 \mathrm{bp}, 440 \mathrm{bp}, 228 \mathrm{bp}, 194 \mathrm{bp}$, and $164 \mathrm{bp}$ corresponded to the CKII gene from -1709 to +85 $(-1709 /+85),-1168 /+85,-441 /+85,-355 /+85,-141 /+85$, $-110 /+85$, and $-80 /+85$, respectively. They were amplified from pT7CKII using forward primers CKII $(-1.7 \mathrm{~K}) \mathrm{F}$, CKII $(-1 \mathrm{~K}) \mathrm{F}$, CKII $(-441) \mathrm{F}, \mathrm{CKII}(-355) \mathrm{F}$, CKII $(-141) \mathrm{F}$, CKII $(-110) \mathrm{F}$, and CKII $(-80) \mathrm{F}$, respectively, and the common reverse primer CKIIR. These resulting PCR products were cloned into pGEM-T Easy Vector (Promega) to produce pT7CKII1.7K, pT7CKII1K, pT7CKII $(-441 /+85), \quad$ pT7CKII $(-355 /+85), \quad$ pT7CKII $(-141 /$ $+85)$, pT7CKII $(-110 /+85)$, and $\operatorname{pT} 7 \mathrm{CKII}(-80 /+85)$, respectively. Then, all plasmids were digested by EcoRI and ligated to EcoRIdigested pDsRed2.1 (Clontech) vectors to generate pCKII1. 
Table 1 Oligonucleotides used in this study

\begin{tabular}{ll}
\hline Symbols & Nucleotide sequences $\left(5^{\prime}-3^{\prime}\right)$ \\
\hline $5^{\prime}$ RACE outer & GCTGATGGCGATGAATGAACACTG \\
CKII-271R & CGAATCCTCCACCACTGCCG \\
$5^{\prime}$ RACE inner & CGCGGATCCGAACACTGCGTTTGCTGGCTTTGATG \\
CKII-117R & GCTGCCCATTGGCACTGCGCTG \\
AP-1 & GTAATACGACTCACTATAGGGC \\
AP-2 & ACTATAGGGCACGCGTGGT \\
CKIIR & CTGTCGTCTACGAGGGGGTGAGG \\
CKII $(-2.3 \mathrm{~K}) \mathrm{F}$ & GTTCGAACAGTGTATTGTAGTTCCTC \\
CKII $(-1.7 \mathrm{~K}) \mathrm{F}$ & GACTGTCAGGAACATTAAAAATCGAC \\
CKII $(-1 \mathrm{~K}) \mathrm{F}$ & GACCAACGGATTAACAATGGGC \\
CKII $(-441) \mathrm{F}$ & GCACTTAAAGCAAACTGAGGGCCC \\
CKII $(-355) \mathrm{F}$ & CTGGTTATGTAAATAAGAGGGGC \\
CKII $(-141) \mathrm{F}$ & GGCGTGTGTATCACTTGGACAGG \\
CKII $(-110) \mathrm{F}$ & GCAGGACAGAAGCCTGAGGC \\
CKII $(-80) \mathrm{F}$ & TTGGCCAGGTGAGCCCCTCCC \\
CKII del $(-141 /-111) \mathrm{F}$ & CAGCAAGATGGCAGGACAGAAGCCTGAGGCAGAAAGGG \\
CKII del $(-141 /-111) \mathrm{R}$ & CAGGCTTCTGTCCTGCCATCTTGCTGGGCTGGTATGGG \\
CKII $(-141 /-111) \mathrm{Fm}$ & AATTGGCGTGTGTATCACTTGGACAAAAAAAAAG \\
CKII $(-141 /-111) \mathrm{Rm}$ & AATTCTTTTTTTTGTCCAAGTGATACACACGCC \\
CKII $(-141 /-111) \mathrm{F}$ & AATTGGCGTGTGTATCACTTGGACAGGGGAAAAAG \\
CKII $(-141 /-111) \mathrm{R}$ & AATTCTTTTCCCCTGTCCAAGTGATACACACGCC \\
\hline
\end{tabular}

AP, adaptor primer; 5'-RACE, 5'-rapid amplification of cDNA end; CK, cytokeratin.

7K-RFP, pCKII1K-RFP, pCKII $(-441 /+85)$-RFP, pCKII $(-355 /$ +85)-RFP, pCKII $(-141 /+85)$-RFP, pCKII $(-110 /+85)$-RFP, and $\mathrm{pCKII}(-80 /+85)$-RFP, respectively. All constructs were confirmed after sequencing.

To generate pCKII $(-560 /+85)-\mathrm{RFP}$, a pT7CKII was digested by EcoRI and HindIII, and a $644 \mathrm{bp}$ fragment was ligated to EcoRI-HindIII-digested pDsRed2.1 (Clontech). To generate pCKII $(-355 /+85) \Delta(-141 /-111)$-RFP, which contains a fragment spanning $-355 /+85$ but with $-141 /-111$ deleted, two PCR products were obtained by amplifying the pT7CKII template using primers of CKIIdel $(-141 /-111) \mathrm{F}$ and CKIIR, and CKII $(-355)$ F and CKIIdel $(-141 /-111)$ R. After two PCR products were annealed, they served as templates for amplification of primers CKII $(-355) \mathrm{F}$ and CKIIR. To generate plasmid pCKII $(-141 /+85)$ KLFm-RFP, which contains a fragment spanning $-141 /+85$ but containing a mutated KLF-binding site $\left({ }^{-119} \mathrm{GGG}^{-117}\right.$ to $\left.{ }^{-119} \mathrm{AAA}^{-117}\right)$, the two PCR products were annealed after they were amplified from the pT7CKII template using primers of CKII $(-141) \mathrm{F}$ and CKII $(-141 /-110) \mathrm{Rm}$, and primers of CKII $(-141 /-110)$ Fm and CKIIR. After annealing, the PCR products served as templates for amplification of the mutated segment by primers CKII( -141$) \mathrm{F}$ and CKIIR. These resulting PCR products were cloned into pGEM-T Easy Vector (Promega) to produce pT7CKII $(-355 /+85) \Delta(-141 /-111)$ and pT7CKII $(-141 /+85)$ KLFm, respectively. Then, pT7CKII $(-355 /$ $+85) \Delta(-141 /-111)$ and pCKII $(-141 /+85)$ KLFm were digested by EcoRI and ligated to EcoRI-digested pDsRed2.1 (Clontech) vectors to generate $\operatorname{pCKII}(-355 /+85) \Delta(-141 /-111)$-RFP and pCKII $(-141 /+85) \mathrm{KLFm}-\mathrm{RFP}$, respectively. To construct plasmid $\operatorname{pEGFPm}(-141 /-111)$, which contained the $-141 /-111$ region of CKII fused with a cytomegalovirus (CMV) minimal TATA promoter and with the enhanced green fluorescent protein (EGFP) reporter gene, primers of CKII $(-141 /-111) \mathrm{F}$ and CKII $(-141 /$ $-111) \mathrm{R}$ were used and the PCR product was inserted into the EcoRI-digested pEGFPm (Chen et al., 2003).

Preparation of plasmids for microinjection

Plasmids pCKII2.3K-RFP, pCKII1.7K-RFP, pCKII1K-RFP, pCKII $(-560 /+85)$-RFP, pCKII $(-441 /+85)-R F P$, pCKII $(-355 /$ $+85)$-RFP,$\quad \operatorname{pCKII}(-141 /+85)$-RFP,$\quad \operatorname{pCKII}(-110 /+85)$-RFP,
pCKII $(-80 /+85)-$ RFP,$\quad p C K I I(-355 /+85) \Delta(-141 /-111)-R F P$ and pCKII $(-141 /+85)$ KLFm-RFP were linearized by XhoI and recovered from a $0.8 \%$ low-melting temperature gel (FMC BioProducts, Philadelphia, PA). DNA intensity and gel absorbancy were measured with a GeneQuant II calculator (Pharmacia Biotech, Hong Kong, China). DNA samples were resuspended at a concentration of $25 \mathrm{ng} / \mu \mathrm{l}$ in double-distilled water mixed with $0.1 \%(\mathrm{v} / \mathrm{v})$ phenol red before use.

To get a quantitative view of the relative strength of the promoter constructs, we classified RFP-expressing embryos into three levels based on the expression pattern in the skin epidermis: strong, medium, and weak. The strong level included embryos displaying RFP expression in approximately $90 \%$ of skin epidermis throughout the body; the medium level included embryos showing RFP expression in $20 \%-90 \%$ of skin epidermis; and the weak level included embryos having RFP-positive signal in less than $20 \%$ of the skin epidermis.

Generation of transgenic germline transmitted zebrafish

The $2.3 \mathrm{~kb}$ pCKII2.3K-RFP was linearized with $X$ hoI and resuspended in $0.1 \mathrm{M} \mathrm{KCl}$ at a final concentration of $50-100 \mathrm{ng} / \mu \mathrm{l}$ with $0.2 \%$ phenol red as a tracer. The DNA solution was microinjected into the cytoplasm of one-cell stage embryos. After microinjection, red fluorescent signal was observed in $24 \mathrm{hr}$ post-fertilization (hpf) embryos. All RFP-positive embryos were raised to adulthood. Pairs of transgenic founder (F0) fish were crossed in a $22 \times 14 \times 13 \mathrm{~cm}$ tank. Parental pairs that produced RFP-positive embryos were separated and mated with wt individuals to confirm the putative germline transmission of the parents. At least 200 embryos per cross were examined for the appearance of red fluorescence. After screening, RFP-positive F1 embryos were raised to adulthood and crossed with wt zebrafish to generate a heterozygotic F2 generation.

Embedding and cryosectioning

The embedding and cryosectioning protocols followed were those of Chen and Tsai (2002), with minor modifications. In brief, embryos were fixed with $4 \%$ paraformaldehyde for $4 \mathrm{hr}$ at $25^{\circ} \mathrm{C}$, dechorionized, mounted with $5 \%$ sucrose containing $1.5 \%$ agarose for $1 \mathrm{hr}$, cut into cubes approximately $5 \times 5 \times 5 \mathrm{~mm}$, and stored in 


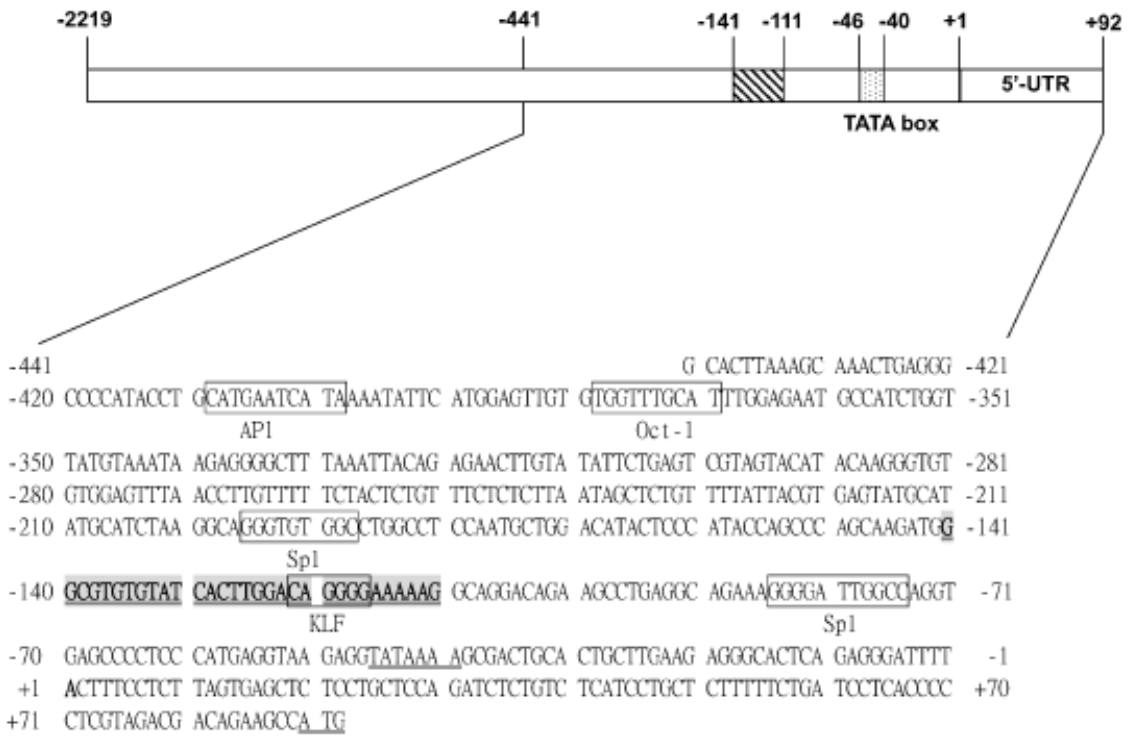

Fig. 1 Schematic representation of sequences in the upstream regions of the zebrafish cytokeratin II (zfCKII) gene. The empty, crossed, and dotted boxes represent the upstream regulatory regions (including $5^{\prime}$-untranslated region, $5^{\prime}$-UTR), the $-141 /-111$ cassette, and the putative TATA box of the zfCKII gene, respectively. The numbers below the boxes indicate the nucleotide positions, with reference to the transcription start site $(+1)$. The detailed sequences of the $-441 /+92$ segment are shown on the lower panel. The putative transcription factors or their binding elements are shown below each corresponding sequence.
$5 \%$ sucrose at $4{ }^{\circ} \mathrm{C}$. All embryos were embedded using Cryomatrix (Shandon, Waltham, MA), and cryosectioning (12 $\mu \mathrm{m}$ thick) was performed with a Microm Cryosector (Heidelberg, Germany).

\section{Microscopy}

Transgenic embryos were observed hourly, especially from 1 to 48 hpf, under a stereo dissecting microscope (MZ12, Leica, Wetzlar, Germany) equipped with a fluorescent module having a GFP or DsRed filter cube (Kramer Scientific, Hampton, NJ). Photographs were taken with an S2 Pro digital camera (Fuji, Tokyo, Japan) when embryos developed at specific stages.

\section{Results}

Upstream regulatory region of the zebrafish CKII gene

Previous studies have shown that zfCKII mRNA is expressed uniformly in the skin, fins, scale epidermis, retina, cornea, neurons, and glial cells of the brain and spinal cord as well as in chondrocytes of the skull of the adult fish (Chua and Lim, 2000). To investigate the molecular mechanism of zfCKII gene expression in epidermal cells, a $2.3 \mathrm{~kb}$ DNA segment of the upstream region of zfCKII was isolated and its partial DNA sequence was determined (Fig. 1; the sequence - 2219/ +92, GenBank Accession No. DQ144236). We also performed a $5^{\prime}$-RACE experiment to determine the transcription start site and the length of the $5^{\prime}$-untranslated region of ZFCKII gene. As shown in Fig. 1, the numbers indicate nucleotide positions with the transcription start site as +1 . Using a string-based search query, the TESS program (http://www.cbil.upenn.edu/ tess/) revealed that the putative binding sites of the transcription factors within the proximal $-441 /+92$ segment were the AP1 site (CATGAATCATA, - 409/ -399), the Oct-1 site (TGGTTTGCAT, - 379/ - 370), two Sp1 sites (GGGTGTGGC, - 196/ - 188; and GGGGATTGGGCC, $-85 /-75$ ), and a TATA box (TATAAAA, $-46 /-40$; Fig. 1).

Transient expression of RFP is driven by the upstream $2.3 \mathrm{~kb}$ segment of the zfCKII gene in zebrafish embryos

The tissue-specific expression mediated by the isolated upstream segment of zfCKII was investigated using a transient transgenic approach. The earliest RFP expression in the embryos microinjected with pCKII2.3KRFP was in the head region at $19 \mathrm{hpf}$ (data not shown). At 3 days post-fertilization (dpf), the RFP signals were extended to the body, including the head (Fig. 2A), skin epidermis, pectoral fins (Fig. 2B), and caudal fins (Fig. 2C). At $14 \mathrm{dpf}$, the red fluorescence was observed in the anal fins and in the retina (Figs. 2D, 2F). These transient expression studies indicated that the upstream segment in the zfCKII $2.3 \mathrm{~K}$ contained regulatory elements that drove RFP expression in a tissue-specific manner.

Functional analysis of the zfCKII regulatory sequence

To determine whether the cis-acting element is sufficient and required for the epidermis-specific expression of zfCKII, serial deletions of an upstream region of zfCKII were generated and fused with RFP cDNA. These constructs were microinjected into zebrafish fertilized eggs. Embryos injected with DNA fragments containing the $-2219,-1709,-1068,-560,-441$, -355 , and $-141 \mathrm{bp}$ upstream sequences [pCKII2.3K-, pCKII1.7K-, pCKII1K-, pCKII $(-560 /+85)-$, pCKII $(-441 /+85)-$, pCKII $(-355 /+85)-$, and pCKII $(-141 /$ $+85)$-RFP, respectively] displayed high RFP expression rates $(63 \%-85 \%$, Table 2) and 100\% RFP-positive 

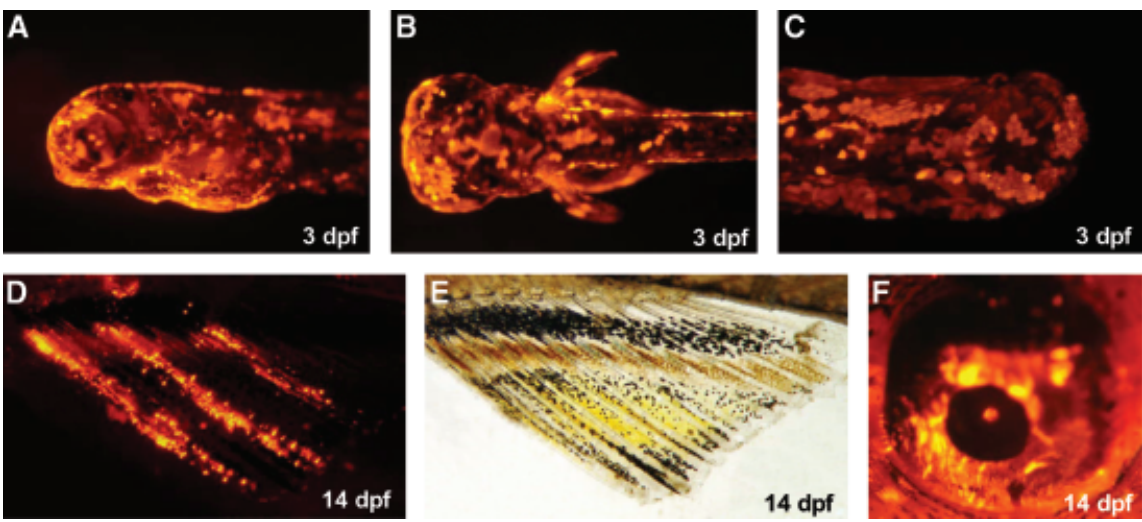

Fig. 2 Transient red fluorescent protein (RFP) expression patterns of the embryos injected with the $2.3 \mathrm{~kb}$ upstream region of the zebrafish cytokeratin II gene. (A) Lateral view of the head region. (B) Dorsal view of the same embryo in A. (C) Lateral view of the caudal region. (D) Lateral view of the anal fin (darkfield). (E) The same embryo as in D, observed under brightfield. (F) Lateral view of the eye. Developmental stages: (A)(C), 3 days post-fertilization (dpf); (D)-(F), $14 \mathrm{dpf}$. signals in the skin epidermis at $3 \mathrm{dpf}$ (Fig. 3A and Table 2). To obtain a quantitative view of the relative strength of the promoter constructs, we classified RFPexpressing embryos into three levels based on the expression pattern in the skin epidermis: strong, medium, and weak with reference to the previous studies $(\mathrm{Ju}$ et al., 2003; Lin et al., 2004). The strong level included embryos displaying RFP expression in approximately $90 \%$ of the skin epidermis throughout the body; the medium level included embryos showing RFP expression in $20 \%-90 \%$ of skin epidermis; and the weak level included embryos having RFP-positive signal in less than $20 \%$ of the skin epidermis (Fig. 3C). As shown in Fig. 3B, embryos displayed relatively high RFP expression rates in strong $(59 \%-78 \%)$ and medium $(20 \%-$ $38 \%$ ) levels when the injected upstream sequences were longer than $-141 \mathrm{bp}$. In addition to red fluorescent skins, embryos injected with DNA fragments containing $-1709,-1068,-560$, and -441 bp upstream sequences also displayed high expression rates $(49 \%-$ $68 \%$ ) of RFP-positive signals in muscle (Table 2; Fig. 3C). However, the pCKII $(-355 /+85)-R F P-$ and pCKII $(-141 /+85)$-RFP-injected embryos displayed red fluorescence in $1.5 \%$ and $3.2 \%$ of muscle fibers (Table 2; Fig. 3C).

It was found that the $-141 \mathrm{bp}$ upstream sequence was able to drive skin-restricted expression of zfCKII. On the other hand, embryos injected with DNA fragments containing less than 110 bp [pCKII $(-110 /+85)$ RFP and pCKII $(-80 /+85)$-RFP] did not give off RFP-positive signals (Fig. 3), even when the concentration of injected DNA was increased to $100 \mathrm{ng} / \mu \mathrm{l}$ (data not shown). On the basis of this evidence, we suggest that the proximal part of the $-141 /+85$ sequence of zfCKII is a minimal cis-element for controlling specific expression.

Cassette $-141 /-111$ was able to direct epidermal cell-specific expression

Interestingly, we found that, when a DNA fragment, in which $-141 /-111$ was deleted from the zfCKII $-355 /+85$ [pCKII $(-355 /+85) \Delta(-141 /-111)-R F P]$, was injected into one-celled embryos, only $1 \%$ (two of 182 , Table 2) of surviving embryos displayed red fluo-

Table 2 Deletion analysis of zfCKII promoter in transient transgenic zebrafish embryos

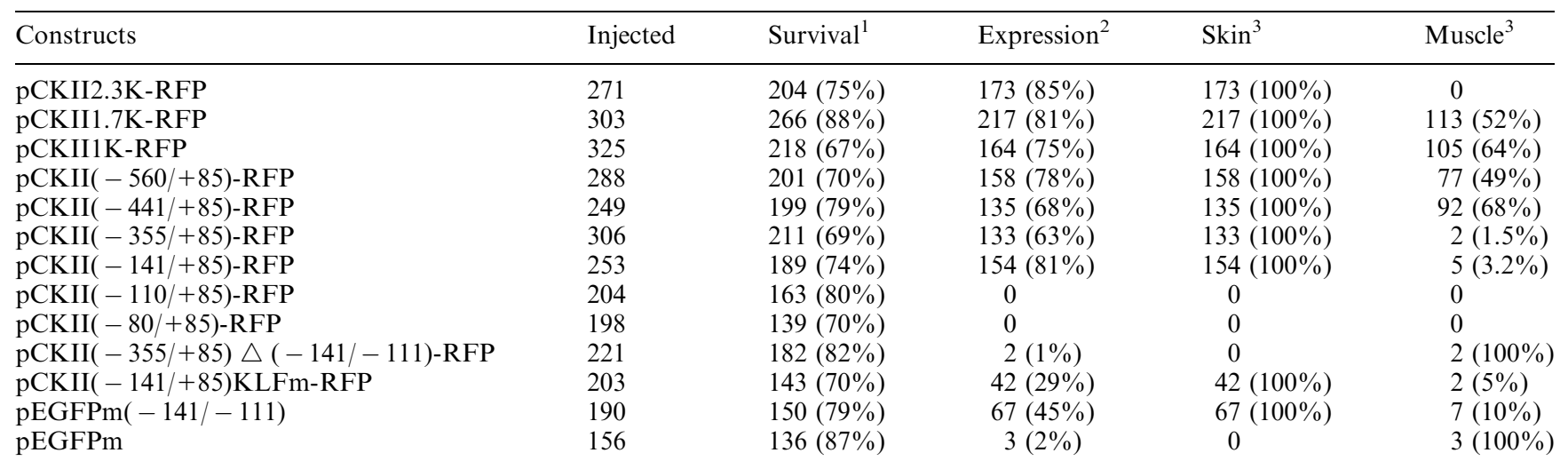

\footnotetext{
${ }^{1}$ Percentages indicate survival rate of injected embryos.

${ }^{2}$ Percentages indicate RFP-expressing embryos among surviving embryos at $3 \mathrm{dpf}$.

${ }^{3}$ Numbers in parentheses represent percentages of RFP-expressing embryos for a particular tissue.

dpf, days post-fertilization; RFP, red fluorescent protein; zfCKII, zebrafish cytokeratin II.
} 
A

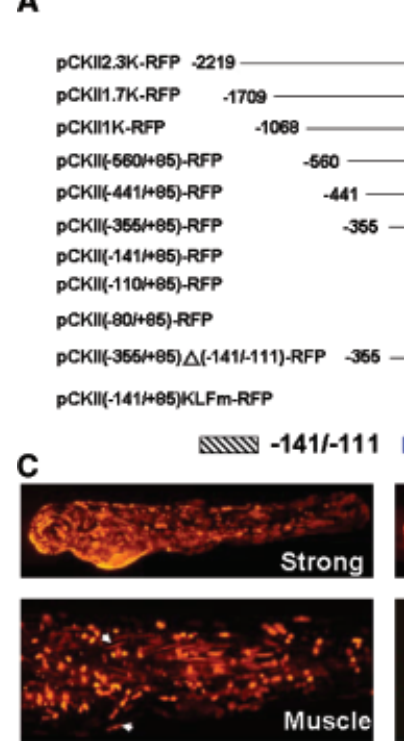

rescence in muscle, and none of the 182 embryos displayed RFP-positive skin epidermis (Table 2). To determine whether cassette $-141 /-111$ was able to direct epidermal cell-specific expression of the reporter gene, we used plasmid $\operatorname{pEGFPm}(-141 /-111)$, in which a mini-promoter of CMV (TATA box only) was fused with GFP and one copy of the $-141 /-111$ cassette (Fig. 4A). Only $2 \%$ of the 136 embryos injected with pEGFPm were GFP-positive, and none had signal detected in the skin (Fig. 4B, left panel; Table 2). However, the expression rate of epidermal cell-specificity in the transgenic embryos injected with pEGFPm $(-141 /$ - 111) was $45 \%$ (Fig. 4B, right panel; Table 2). Furthermore, a mutation plasmid, pCKII $(-141 /+85)$ KLFm-RFP, in which ${ }^{-119} \mathrm{GGG}^{-117}$ was mutated to ${ }^{-119} \mathrm{AAA}^{-117}$, was injected into the embryos and revealed a $29 \%$ RFP expression rate (Table 2). Interestingly, most of the pCKII $(-141 /+85) \mathrm{KLFm}-\mathrm{RFP}$ injected embryos displayed medium $(43 \%)$ and weak $(52 \%)$ RFP expression, and only $5 \%$ displayed strong RFP expression (Fig. 3B). Based on these observations, we propose that cassette $-141 /-111$ was able to direct epidermal cell-specific expression and that ${ }^{-119} \mathrm{GGG}^{-117}$ is an important element within cassette $-141 /-111$.

RFP expression in germline zfCKII-RFP transgenic zebrafish

To generate stable transgenic lines for further analysis of the expression mechanism of zfCKII, embryos injected with pCKII2.3K-RFP and exhibiting RFP expression were collected and raised to adulthood. Founder fishes (F0) were crossed with wt or crossed with each other to generate $\mathrm{F} 1$ embryos. Of 63 founder fish tested, one line, $\operatorname{Tg}(\mathrm{zfCKII}(2.3)$ :GFP), produced embryos that expressed RFP in the epidermis, but $5 \%$
(11 positive of 234) of embryos expressed RFP at $24 \mathrm{hpf}$, suggesting that the transgenic founder is a germline mosaic, which is a common finding in transgenic fish at the founder generation (reviewed by Gong and Hew, 1995). The F2 inheritance rate of RFP-positive F1 individuals of the $\operatorname{Tg}(\mathrm{zfCKII}(2.3)$ : RFP $)$ line is $48.57 \%$ (102 of 210 total embryos), indicating that there was a single insertion site of the transgene in the genome.

All F2 embryos (154 of 154 embryos) derived from an F1 female crossed with a wt male exhibited red fluorescent signal at one-cell (Fig. 5A), blastula (Fig. 5B), and segmentation (Fig. 5C) stages. Red fluorescence became stronger and extended to the body at $3 \mathrm{dpf}$ (Fig. 5D). In contrast, none of the F2 embryos derived from an F1 male crossed with a wt female showed red fluorescence at the one-cell (Fig. 5E) and blastula stages (Fig. 5F), yet $46.9 \%$ of 145 embryos began to display red fluorescence at $14 \mathrm{hpf}$ (data not shown). The red fluorescence became stronger at $24 \mathrm{hpf}$ (Fig. 5G), and extended to the trunk in a non-uniform expression manner (Fig. 5H). This evidence strongly demonstrates that the zfCKII transcripts are maternally inherited.

Cryosectioning of $4 \mathrm{dpf}$ embryos derived from the $\operatorname{Tg}(z f C K I I(2.3): R F P)$ line revealed RFP signal distributed in the retina (Fig 5I), head epidermis (Fig. 5I), trunk epidermis (Fig. 5K), and pectoral fins (Fig. 5K). In addition, we think the faint red fluorescent signal in the brain in the $4 \mathrm{dpf}$ larva section (Fig. 5I) represents real signals reflecting the endogenous gene expression. In the 1-month-old juveniles, red fluorescence was also observed on the body surface, on the cornea (Fig. 5M), and in the epidermal cells of the scales (Fig. 5N). This red fluorescence was found only in the outer region of the scale (Fig. 5O). From these observations, we conclude that $\mathrm{Tg}(\mathrm{zFCKII}(2.3)$ :RFP) fish recapitulate the endogenous zfCKII expression patterns. 


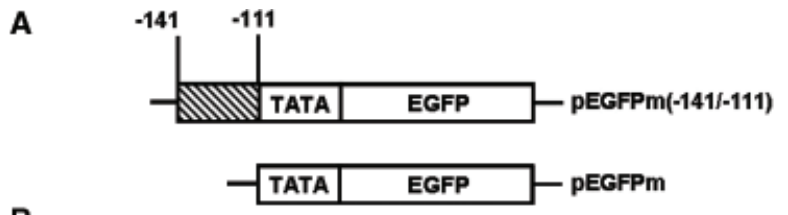

B

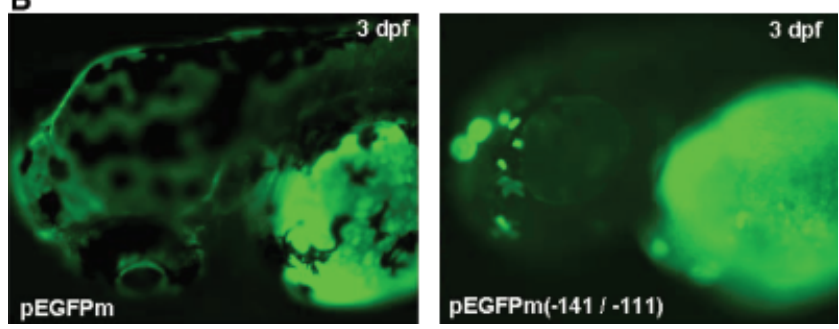

Fig. 4 Cassette $-141 /-111$ is able to direct skin-specific expression of the cytomegalovirus (CMV) basal promoter. (A) Plasmids pEGFPm and pEGFPm $(-141 /-111)$, including a minimal TATA box derived from a CMV promoter fused with the enhanced green fluorescent protein (EGFP) gene and one copy of cassette $-141 /-111$ fused with pEGFPm, respectively. (B) Embryos were photographed under blue light equipped with a green fluorescent protein (GFP) filter. In pEGFPm- (left panel) and pEGFPm $(-141 /-111)$-injected (right panel) zebrafish, enhanced GFP (EGFP) signals appeared in the skin epidermis. The uniform green staining seen in the embryo injected with the pEGFPm control construct (left panel) is due to the auto-fluorescence of the embryo. The fluorescence was checked and observed when the embryos developed at 3 days post-fertilization (dpf).

\section{Discussion}

In this study, we isolated a $2.3 \mathrm{~kb}$ upstream regulatory sequence of the zfCKII gene. Analyses of transient as well as stable transgenic lines revealed that this $2.3 \mathrm{~kb}$ segment is able to recapitulate the endogenous zfCKII expression patterns. Deletion analyses were performed and showed that a proximal $-141 /+85$ sequence of zfCKII is a minimal cis-element for controlling expression specificity. Finally, we identified a 31 bp $(-141 /$ -111) segment, which is able to drive GFP expression in the skin epidermis.

Many transcription factors (c-jun, c-fos, C/EBP, and SP-1) and their binding sites (AP1, CCAAT-, and GCbox) have been reported to play important roles in regulating mammal keratin genes at transcription (Brembeck and Rustgi, 2000; Sterneck et al., 2006; Wang et al., 2006). As shown in Fig. 1, AP1, CCAAT-, and GC-box exist in the $-441 /+85$ segment of the zfCKII gene. We examined the putative transcription factor-binding sites in zebrafish $\mathrm{k} 8$ (now renamed as $\mathrm{k} 4$ ) and k18 genes (Gong et al., 2002; Wang et al., 2006) and found that AP1, CCAAT-, and GC-box are located in the upstream regions of $\mathrm{k} 4$ and $\mathrm{k} 18$. Thus, we propose that zebrafish keratin genes zfCKII, k4, and k18 shared some common transcriptional regulatory mechanisms with those of keratin genes in mammals.

Systematic analyses of mammal keratin revealed that the tissue specificity is most probably conferred by sequences close to the TATA box. But in some cases, the enhancers for directing tissue specificity are located at the first intron (Rhodes and Oshima, 1998) and the $3^{\prime}$ flanking sequence (Hu and Gudas, 1994). A transgenic analysis of the human $\mathrm{k} 5$ promoter revealed that $90 \mathrm{bp}$ of the $5^{\prime}$-flanking sequences contain sufficient information to direct expression to keratinocytes (Byrne and Fuchs, 1993). Mahony et al. (2000) reported that a
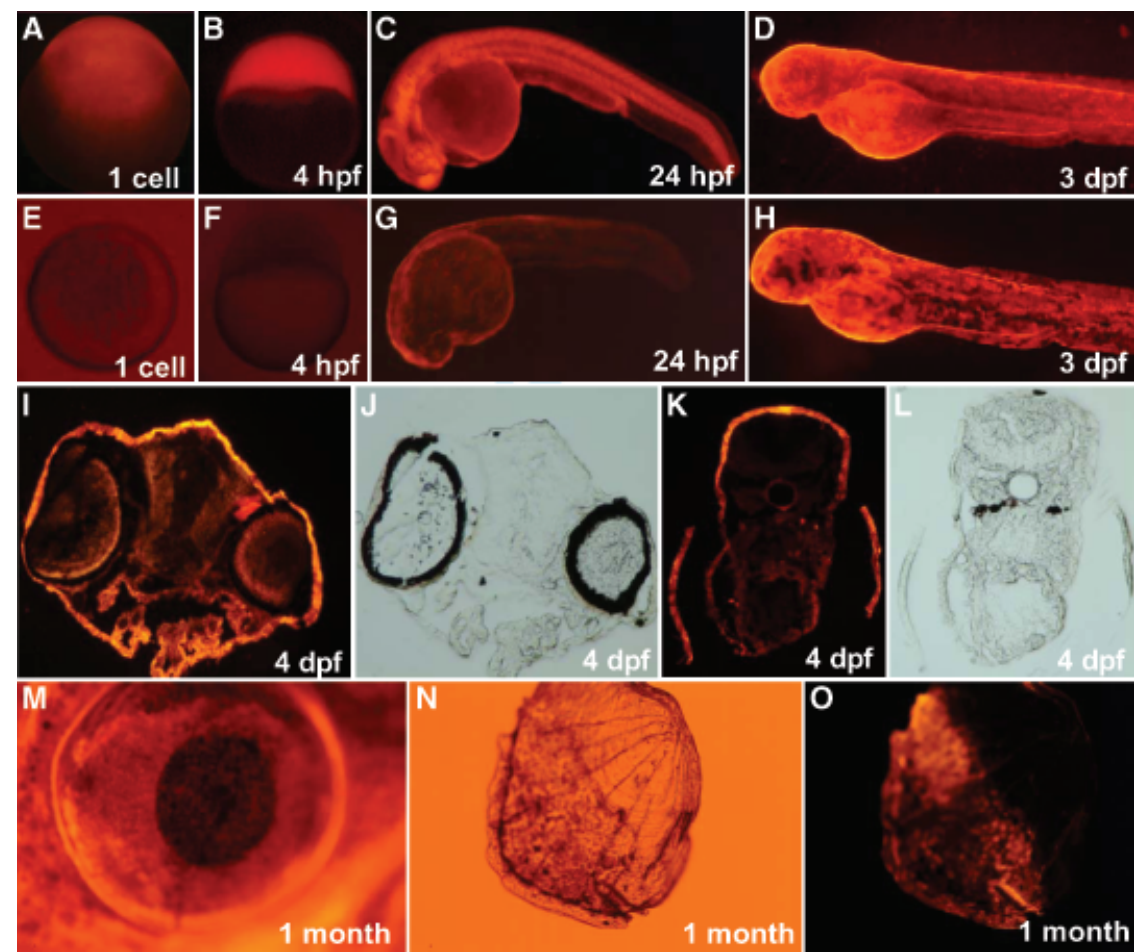

Fig. 5 Red fluorescent protein (RFP) expression in transgenic fish lines. F2 offspring were produced by mating $\mathrm{F} 1$ females from the $\operatorname{Tg}(z f C K I I(2.3)$ :RFP) line with wild-type (wt) males (A-D) or by mating the F1 males from the $\operatorname{Tg}(\mathrm{zfCKII}(2.3): \mathrm{RFP})$ line with wt females $(\mathbf{E}-\mathbf{H})$. (A, E) At the one-cell stage. (B, F) At $4 \mathrm{hr}$ post-fertilization (hpf). (C, G) At $24 \mathrm{hpf}$. (D, H) At 3 days post-fertilization (dpf). (I) Cross-section of the head region at $4 \mathrm{dpf}$. (J) Brightfield observation of I. (K) Cross-section of the trunk region at $4 \mathrm{dpf}$. (L) Brightfield observation of K. (M) Eye of a 1-month-old juvenile. (N) A scale of a 1-month-old juvenile. (O) Darkfield observation. zfCKII, zebrafish cytokeratin II. 
$120 \mathrm{bp}$ mouse keratin $6 \mathrm{a}$ mini-gene contains sufficient sequence information to direct uniform and tissuespecific expression. In zfCKII, we showed that the proximal $-141 /+85$ sequence of zfCKII is a minimal cis-element and that a 31 bp $(-141 /-111)$ segment is capable of driving GFP expression in the skin epidermis. Sequence analysis of $-141 /-111$ segment revealed that there is one $\mathrm{KLF}$-binding site $\left({ }^{-122} \mathrm{CA}\right.$ $\mathrm{GGGG}^{-117}$ ). KLF has been reported to be involved in regulating tissue-dependent transcription of the keratin 19 gene (Brembeck and Rustgi, 2000). We have shown a mutation plasmid, pCKII $(-141 /+85) K L F m-R F P$, in which ${ }^{-119} \mathrm{GGG}^{-117}$ has been mutated to ${ }^{-119}$ $\mathrm{AAA}^{-117}$, resulting in an altered KLF-binding site. Comparing the pCKII $(-141 /+85)$-RFP- with the pCKII $(-141 /+85)$ KLFm-RFP-injected groups, we found that the RFP expression rates were significantly reduced ( $81 \%$ versus $29 \%$, respectively; Table 2). Only $5 \%$ of pCKII $(-141 /+85) \mathrm{KLFm}$-RFP-injected embryos displayed strong RFP expression, a rate much lower than that of the pCKII $(-141 /+85)$-RFP-injected group (78\%, Fig. 3B). These observations strongly suggest that ${ }^{-122} \mathrm{CAGGGG}^{-117}$ might be an enhancer for the zfCKII gene.

Embryos injected with pCKII2.3K-RFP displayed normal skin-specific expression (Table 2). However, embryos injected with pCKII1.7K-, pCKII1K-, pCKII $(-560$ / $+85)-, \quad$ pCKII $(-441 /+85)-, \quad$ pCKII $(-355 /+85)-$, and pCKII $(-141 /+85)$-RFP displayed ectopic expression in muscle (Table 2). Thus, we propose that there is a muscle repressor in the -2.3 to $-1.7 \mathrm{~kb}$ region.

Chua and Lim (2000) showed that zfCKII mRNA was inherited maternally. The mechanism of transcriptional regulation of maternally inherited genes is difficult to study because of the interference of maternally produced mRNA. Here, for the first time, we demonstrate that an upstream $2.3 \mathrm{~kb}$ segment of the zfCKII gene is able to drive zfCKII expression before mid-blastula transition and is sufficient to recapitulate the endogenous zfCKII transcription. We used the $\operatorname{Tg}(\mathrm{zfCKII}(2.3)$ :RFP) line for studying the regulatory mechanism. F2 embryos derived from $\operatorname{Tg}(\mathrm{zfCKII}(2.3)$ : RFP) males crossed with wt females displayed red fluorescence without an interfering maternal effect. Thus, this fish line can be used as an excellent tool for studying zygotic expression of maternally inherited genes. In fact, we can knock-down several putative transcription factors, such as KLF, c-Jun/Fos, or SP1, by injecting the morpholinos into the $\mathrm{F} 2$ embryos that were produced by mating $\operatorname{Tg}(z f C K I I(2.3): R F P)$ males with wt females to study the zygotic regulation of zfCKII. This transgenic line should provide new insights into zfCKII expression at the transcription level in early embryogenesis.

Acknowledgments This work was supported by the National Science Council, Republic of China, under Grant numbers NSC 93-
2313-B-032-003 (Y. H. C.), AOC 93-9.2.4-FI(2), and NSC 91-2811B-002-029 (H. J. T.).

\section{References}

Ainis, L., Tagliafierro, G., Mauceri, A., Licata, A., Ricca, M.B. and Fasulo, S. (1995) Cytokeratin type distribution in the skin and gill epithelia of the Indian fresh-water catfish, Heteropenustes fossilis as detected by immunohistochemistry. Folia Histochem Cytobiol 33:77-81.

Arenas, M.I., Fraile, B., de Miguel, M. and Paniagua, R. (1995) Intermediate filaments in the testis of the teleost mosquito fish (Gambusia affinis holbrooki): a light and electron microscopy immunocytochemical study and western blotting analysis. Histochem J 27:329-337.

Brembeck, F.H. and Rustgi, A.K. (2000) The tissue-dependent keratin 19 gene transcription is regulated by GKLF/KLF4 and Sp1. J Biol Chem 275:28230-28239.

Byrne, C. and Fuchs, E. (1993) Probing keratinocyte and differentiation specificity of the human $\mathrm{K} 5$ promoter in vitro and in transgenic mice. Mol Cell Biol 13:3176-3190.

Byrne, C., Tainsky, M. and Fuchs, E. (1994) Programming gene expression in developing epidermis. Development 120:2369-2383.

Chen, Y.H., Lee, H.C., Liu, C.F., Lin, C.Y. and Tsai, H.J. (2003) Novel regulatory sequence $-82 /-62$ functions as a key element to drive the somite-specificity of zebrafish $m y f-5$. Dev Dyn 228:41-50.

Chen, Y.H., Lee, W.C., Liu, C.F. and Tsai, H.J. (2001) Molecular structure, dynamic expression and promoter analysis of zebrafish (Danio rerio) myf-5 gene. Genesis 29:22-35.

Chen, Y.H. and Tsai, H.J. (2002) Treatment with myf5-morpholino results in somite patterning and brain formation defects in zebrafish. Differentiation 70:447-456.

Chua, K.L. and Lim, T.M. (2000) Type I and type II cytokeratin cDNAs from the zebrafish (Danio rerio) and expression patterns during early development. Differentiation 66:31-41.

Conrad, M., Lemb, K., Schubert, T. and Markl, J. (1998) Biochemical identification and tissue-specific expression patterns of keratins in the zebrafish (Danio rerio). Cell Tissue Res 293:195-205.

Druger, R.K., Levine, E.M., Glasgow, E., Jones, P.S. and Schechter, N. (1992) Cloning of a type I keratin from goldfish optic nerve: differential expression of keratins during regeneration. Differentiation 52:33-43.

Fuchs, E. and Weber, K. (1994) Intermediate filaments: structure, dynamics, function, and disease. Annu Rev Biochem 63: 345-382.

Giordano, S., Glasgow, E., Tesser, P. and Schechter, N. (1989) A type II keratin is expressed in glial cells of the goldfish visual pathway. Neuron 2:1507-1516.

Giordano, S., Hall, C., Quitschke, W., Glasgow, E. and Schechter, N. (1990) Keratin 8 of simple epithelia is expressed in glia of the goldfish nervous system. Differentiation 44:163-172.

Gong, Z. and Hew, C.L. (1995) Transgenic fish in aquaculture and developmental biology. Curr Top Dev Biol 30:177-214.

Gong, Z., Ju, B., Wang, X., He, J., Wan, H., Sudha, P.M. and Yan, T. (2002) Green fluorescent protein expression in germ-line transmitted transgenic zebrafish under a stratified epithelial promoter from keratin 8. Dev Dyn 223:204-215.

Groff, J.M., Naydan, D.K., Higgins, R.J. and Zinkl, J.G. (1997) Cytokeratin-filament expression in epithelial and non-epithelial tissues of the common carp (Cyprinus carpio). Cell Tissue Res 287:375-384.

Hatzfeld, M., Maier, G. and Franke, W.W. (1987) Cytokeratin domains involved in heterotypic complex formation determined by in-vitro binding assays. J Mol Biol 197:237-255.

Hu, L. and Gudas, L.J. (1994) Activation of keratin 19 gene expression by a $3^{\prime}$ enhancer containing an AP1 site. J Biol Chem 269:183-191. 
Imboden, M., Goblet, C., Korn, H. and Vriz, S. (1997) Cytokeratin 8 is a suitable epidermal marker during zebrafish development. $\mathrm{C}$ R Acad Sci III 320:689-700.

Ju, B., Chong, S.W., He, J., Wang, X., Xu, Y., Wan, H., Tong, Y., Yan, T., Korzh, V. and Gong, Z. (2003) Recapitulation of fast skeletal muscle development in zebrafish by transgenic expression of GFP under the mylz2 promoter. Dev Dyn 227: 14-26.

Kimmel, C.B., Ballard, W.W., Kimmel, S.R., Ullmann, B. and Schilling, T.F. (1995) Stages of embryonic development of the zebrafish. Dev Dyn 203:253-310.

Klymkowsky, M. (1995) Intermediate filaments: new proteins, some answers, more questions. Curr Opin Cell Biol 7:46-54.

Leask, A., Rosenberg, M., Vassar, R. and Fuchs, E. (1990) Regulation of a human epidermal keratin gene: sequences and nuclear factors involved in keratinocyte-specific transcription. Genes Dev 4:1985-1998.

Lin, C.Y., Chen, Y.H., Lee, H.C. and Tsai, H.J. (2004) A novel ciselement in intron 1 represses somite expression of zebrafish myf5. Gene 334:63-72.

Mahony, D., Karunaratne, S., Cam, G. and Rothnagel, J.A. (2000) Analysis of mouse keratin 6a regulatory sequences in transgenic mice reveals constitutive, tissue-specific expression by a keratin 6a minigene. J Invest Dermatol 115:795-804.

Markl, J. and Franke, W.W. (1988) Localization of cytokeratins in tissues of the rainbow trout: fundamental differences in expression pattern between fish and higher vertebrates. Differentiation 39:97-122.

Markl, J., Winter, S. and Franke, W.W. (1989) The catalog and the expression complexity of cytokeratins in a teleost fish, the rainbow trout. Eur J Cell Biol 50:1-16.

Popa, C., Dahler, A.L., Serewko-Auret, M.M., Wong, C.F., Smith, L., Barnes, L.M., Strutton, G.M. and Saunders, A.N. (2004) AP2 transcription factor family member expression, activity, and regulation in human epidermal keratinocytes in vitro. Differentiation 72:185-197.

Rhodes, K. and Oshima, R.G. (1998) A regulatory element of the human keratin 18 gene with AP-1-dependent promoter activity. J Biol Chem 273:26534-26542.
Rogers, A.M., Winter, H., Langbein, L., Bleiler, R. and Schweizer, J. (2004) The human type I keratin gene family: characterization of new hair follicle specific members and evaluation of the chromosome 17q21.2 gene domain. Differentiation 72:527-540.

Schaffeld, M., Haberkamp, M., Braziulis, E., Lieb, B. and Markl, J. (2002a) Type II keratin cDNAs from the rainbow trout: implications for keratin evolution. Differentiation 70:292-299.

Schaffeld, M., Hoffling, S., Haberkamp, M., Conrad, M. and Markl, J. (2002b) Type I keratin cDNAs from the rainbow trout: independent radiation of keratins in fish. Differentiation 70:282-291.

Schaffeld, M., Knappe, M., Hunzinger, C. and Markl, J. (2003) cDNA sequences of the authentic keratins 8 and 18 in zebrafish. Differentiation 71:73-82.

Schaffeld, M., Lobbecke, A., Lieb, B. and Markl, J. (1998) Tracing keratin evolution: catalog, expression patterns and primary structure of shark (Scyliorhinus stellaris) keratins. Eur J Cell Biol 77:69-80.

Sterneck, E., Zhu, S., Jorcano, J.L. and Smart, R.C. (2006) Conditional ablation of C/EBPbeta demonstrates its keratinocyte-specific requirement for cell survival and mouse skin tumorigenesis. Oncogene 25:1272-1276.

Vassar, R., Rosenberg, M., Ross, S., Tyner, A. and Fuchs, E. (1989) Tissue-specific and differentiation-specific expression of a human K14 keratin gene in transgenic mice. Proc Natl Acad Sci USA 86:1563-1567.

Wang, Y.N., Chen, Y.J. and Chang, W.C. (2006a) Activation of ERK signaling by epidermal growth factor mediates c-Jun activation and p300 recruitment in keratin 16 gene expression. Mol Pharmacol 69:85-98.

Wang, Y.H., Chen, Y.H., Lin, Y.J. and Tsai, H.J. (2006b) Spatiotemporal expression of zebrafish keratin 18 during early embryogenesis and the establishment of a keratin 18:RFP transgenic line. Gene Expr Patterns 6:335-339.

Westerfield, M. (1995) The Zebrafish Book. 3rd ed. University of Oregon Press, Eugene.

Zaccone, G., Howi, A.J., Mauceri, A., Fasulo, S., Lo Casio, P. and Youson, J.H. (1995) Distribution patterns of cytokeratins in epidermis and horny teeth of the adult sea lamprey, Petromyzon marinus. Folia Histochem Cytobiol 33:69-75. 